\title{
Generating function, path integral representation, and equivalence for stochastic exclusive particle systems
}

\author{
Su-Chan Park \\ School of Physics, Korea Institute for Advanced Study, Seoul 130-722, Korea \\ Jeong-Man Park \\ Department of Physics, The Catholic University of Korea, Puchon 420-743, Korea
}

\begin{abstract}
We present the path integral representation of the generating function for classical exclusive particle systems. By introducing hard-core bosonic creation and annihilation operators and appropriate commutation relations, we construct the Fock space structure. Using the state vector, the generating function is defined and the master equation of the system is transformed into the equation for the generating function. Finally, the solution of the linear equation for the generating function is derived in the form of the path integral. Applying the formalism, the equivalence of reaction-diffusion processes of single species and two species is described.
\end{abstract}

PACS numbers: 05.70.Ln 


\section{INTRODUCTION}

Recently, nonequilibrium systems such as reaction-diffusion systems, driven lattice systems, and cellular automata have been intensely investigated, not only because of their connections to a variety of important physical phenomena (nonequilibrium phase transitions, long-range correlations, and self-organized criticality), but also because of the analytic challenge due to the lack of a general formalism to describe nonequilibrium systems [1]. Although numerical approaches have played a major role in the investigation of nonequilibrium systems and have been successful in many areas, a general field-theoretic formalism has been constantly sought because, along with a renormalization-group analysis, it would provide a systematic tool to evaluate physical observables in the scaling regime 2]. It is difficult to investigate nonequilibrium systems analytically at the microscopic scale because there are too many microscopic variables involved, and microscopic variables take discrete values. A field-theoretic strategy is to find, starting from the microscopic rules, the equations of motion of well-chosen coarse-grained variables. These mesoscopic variables take continuous values and vary continuously in space and time. They describe the system at the mesoscopic scale, while keeping track of all the fluctuations related to the microscopic degrees of freedom. Doi first introduced a field-theoretic formalism for reaction-diffusion systems using the bosonic coherent state path integral [3], and several authors revived the formalism and incorporated renormalization-group approaches to the description of the anomalous kinetics in reaction-diffusion systems and the stochastic sandpile model [4, 5, 6, 7, 8, 9$]$.

Despite the success achieved by the bosonic field theory for reaction-diffusion systems, some nonequilibrium systems with exclusive particles cannot be analyzed correctly by the bosonic field theory. Driven reaction-diffusion systems [10, 11], multispecies adsorption models [12], and driven lattice gases (DLG) [13] are some examples to which the bosonic field theory cannot be applied. In these systems, the exclusive property of the particles is important. Several attempts have been made to incorporate the exclusive property into a field theory. One way to take the exclusive property into account is to exploit the physical knowledge of the system phenomenologically as Zia and Schmittmann [13] have done for the DLG. This method is an approximation and cannot be systematic. Other approaches proposed by Brunel et al. [14] and Bares and Mobilia [15] are formulated using the fermionic path integral and are rather difficult to analyze and to extend for higher spatial dimen- 
sions or multispecies processes. Recently, van Wijland extended the bosonic field theory by introducing the exclusion constraint operator to take the exclusive property into account 16]. He used bosonic creation and annihilation operators to construct the path integral by considering the exclusive property using the exclusion constraint operator.

The authors previously presented a hard-core bosonic field theory using hard-core bosonic creation and annihilation operators [17]. It has been successfully applied to the asymmetric exclusion process and several restricted solid-on-solid-type growth models to provide the correct Langevin-type equations of motion and the proper path integral formulation [18]. However, the formalism assumed the existence of the Fokker-Planck equations for processes a priori and derived the Langevin type equations of motion. In this paper, we develop a more general path integral formalism for nonequilibrium systems with exclusive particles. In what follows, we present the derivation of the path integral formulation for systems with exclusive particles using the generating function of the correlation functions, and we will illustrate how the formalism can be applied to various reaction-diffusion systems to establish nonequilibrium universality classes.

\section{FORMALISM}

The dynamics of a stochastic system are usually described by the master equation governing the time evolution of the probability $P(\mathcal{C} ; t) . P(\mathcal{C} ; t)$ is the probability of a system being in a microstate $\mathcal{C}$ at time $t$. The master equation takes the form [19, 20, 21]

$$
\frac{\partial}{\partial t} P(\mathcal{C} ; t)=\sum_{\mathcal{C}^{\prime} \neq \mathcal{C}}\left\{W_{\mathcal{C C}^{\prime}} P\left(\mathcal{C}^{\prime} ; t\right)-W_{\mathcal{C}^{\prime} \mathcal{C}} P(\mathcal{C} ; t)\right\},
$$

where $W_{\mathcal{C}^{\prime} \mathcal{C}}$ is the transition rate, or transition probability per unit time, from microstate $\mathcal{C}$ to $\mathcal{C}^{\prime}$. Introducing the matrix $H_{\mathcal{C C}^{\prime}}$,

$$
H_{\mathcal{C C}^{\prime}}=\delta_{\mathcal{C C}^{\prime}} \sum_{\mathcal{C}^{\prime \prime}} W_{\mathcal{C}^{\prime \prime} \mathcal{C}}-W_{\mathcal{C} C^{\prime}}
$$

Eq. (11) can be written succinctly as

$$
\frac{\partial}{\partial t} P(\mathcal{C} ; t)=-\sum_{\mathcal{C}^{\prime}} H_{\mathcal{C}^{\prime}} P\left(\mathcal{C}^{\prime} ; t\right) .
$$

Due to the resemblance of the master equation to the Schrödinger equation, it is convenient to introduce Dirac's bra-ket notation and an orthonormal basis formed by microstates $\{|\mathcal{C}\rangle\}$. 
Introducing the state vector $|P ; t\rangle \equiv \sum_{\mathcal{C}} P(\mathcal{C} ; t)|\mathcal{C}\rangle$ and the Hamiltonian operator $\hat{H}$ whose component is $H_{\mathcal{C C}^{\prime}}$ in this orthonormal basis, the master equation can be written in the form of an imaginary time Schrödinger equation,

$$
\frac{\partial}{\partial t}|P ; t\rangle=-\hat{H}|P ; t\rangle .
$$

In this representation, the average of a physical quantity can be written as

$$
\langle\mathcal{O}\rangle=\sum_{\mathcal{C}} \mathcal{O}(\mathcal{C}) P(\mathcal{C} ; t)=\langle\cdot|\hat{\mathcal{O}}| P ; t\rangle,
$$

where $\hat{\mathcal{O}}$ is an operator with the elements $\left\langle\mathcal{C}^{\prime}|\hat{\mathcal{O}}| \mathcal{C}\right\rangle=\delta_{\mathcal{C}, \mathcal{C}^{\prime}} \mathcal{O}(\mathcal{C})$ and the projection state $\langle\cdot|$ is defined as

$$
\langle\cdot| \equiv \sum_{\mathcal{C}}\langle\mathcal{C}| .
$$

A natural way to deal with a stochastic system in which the particle number at each site varies is to introduce a Fock-space-like structure. When a system has $M$ different particle species with the exclusive property, at each site there are $(M+1)$ possible states: a vacuum state and $M$ occupied states depending on particle species. Using Greek indices for the kinds of particle species - a vacuum state is regarded as a new species and 0 is reserved for a vacuum state - and bold Latin indices for site locations, we denote a state at the site $\boldsymbol{n}$ by $\left|\alpha_{\boldsymbol{n}}\right\rangle$, where $\alpha_{\boldsymbol{n}}$ is the index of species occupying the site $\boldsymbol{n}$ and goes from 0 to $M$. For microstates of the whole system, we work in a phase space which is composed of the direct product of single-site microstates of all sites $\{\boldsymbol{n}\}$,

$$
|\{N\}\rangle=\bigotimes_{n}\left|\alpha_{n}\right\rangle .
$$

At each site $\boldsymbol{n}$, creation and annihilation of the exclusive particles are described by creation and annihilation operators as follows:

$$
\hat{a}_{\alpha, \boldsymbol{n}}=\left|0_{\boldsymbol{n}}\right\rangle\left\langle\alpha_{\boldsymbol{n}}\left|, \quad \hat{a}_{\alpha, \boldsymbol{n}}^{\dagger}=\right| \alpha_{\boldsymbol{n}}\right\rangle\left\langle 0_{\boldsymbol{n}}\right| \quad(\alpha \neq 0),
$$

with the closure relation

$$
\sum_{\alpha=0}^{M}\left|\alpha_{n}\right\rangle\left\langle\alpha_{n}\right|=I
$$

and the orthonormality condition

$$
\left\langle\alpha_{\boldsymbol{n}} \mid \beta_{\boldsymbol{n}}\right\rangle=\delta_{\alpha \beta}, \quad \alpha, \beta=0,1, \ldots, M .
$$


These operators obey the hard-core bosonic commutation relations

$$
\begin{aligned}
\hat{a}_{\alpha, \boldsymbol{n}} \hat{a}_{\beta, \boldsymbol{n}} & =\hat{a}_{\alpha, \boldsymbol{n}}^{\dagger} \hat{a}_{\beta, \boldsymbol{n}}^{\dagger}=0, \\
\hat{a}_{\alpha, \boldsymbol{n}} \hat{a}_{\alpha, \boldsymbol{n}}^{\dagger} & =1-\sum_{\gamma=1}^{M} \hat{a}_{\gamma, \boldsymbol{n}}^{\dagger} \hat{a}_{\gamma, \boldsymbol{n}}, \\
{\left[\hat{a}_{\alpha, \boldsymbol{n}}, \hat{a}_{\beta, \boldsymbol{m}}^{\dagger}\right] } & =\left[\hat{a}_{\alpha, \boldsymbol{n}}, \hat{a}_{\beta, \boldsymbol{m}}\right]=0 \quad(\boldsymbol{n} \neq \boldsymbol{m}),
\end{aligned}
$$

where Eqs. (11a) and (11b) prohibit double occupancy at a single site even for different species - hence the nomenclature "hard core." Equation (11c) states that any two operators defined at different sites commute-hence the name "boson." As usual, $\hat{N}_{\alpha, \boldsymbol{n}}=\hat{a}_{\alpha, \boldsymbol{n}}^{\dagger} \hat{a}_{\alpha, \boldsymbol{n}}$ is the number operator whose eigenvalues are 0 (vacant) and 1 (occupied).

To each state of the system, we can associate the state vector $|P ; t\rangle$, considered an element of Fock space,

$$
|P ; t\rangle=\sum_{\{N\}} P(\{N\} ; t)|\{N\}\rangle,
$$

where $|\{N\}\rangle$ is the microstate defined in Eq. (7). The microstate can be written in terms of hard-core boson operators as

$$
\begin{aligned}
& |\{N\}\rangle=\prod_{\boldsymbol{n}} \prod_{\alpha=1}^{M}\left(\hat{a}_{\alpha, \boldsymbol{n}}^{\dagger}\right)^{N_{\alpha, \boldsymbol{n}}}|\{0\}\rangle, \\
& \langle\{N\}|=\langle\{0\}| \prod_{\boldsymbol{n}} \prod_{\alpha=1}^{M}\left(\hat{a}_{\alpha, \boldsymbol{n}}\right)^{N_{\alpha, \boldsymbol{n}},}
\end{aligned}
$$

where $N_{\alpha, \boldsymbol{n}}$ is the eigenvalue of the number operator corresponding to the eigenstate $\left.\{N\}\right\rangle$ $\left(\hat{N}_{\alpha, \boldsymbol{n}}|\{N\}\rangle=N_{\alpha, \boldsymbol{n}}|\{N\}\rangle\right)$. Using hard-core boson operators, the projection state can be written as

$$
\langle\cdot|=\langle\{0\}| \prod_{\boldsymbol{n}}\left(1+\sum_{\alpha=1}^{M} \hat{a}_{\alpha, \boldsymbol{n}}\right) .
$$

Now we introduce the generating function $F(\{\bar{\varphi}\} ; t)$, defined as

$$
F(\{\bar{\varphi}\} ; t)=\langle\{\bar{\varphi}\} \mid P ; t\rangle,
$$

where

$$
\begin{aligned}
\langle\{\bar{\varphi}\}| & \equiv \bigotimes_{\boldsymbol{n}}\left(\left\langle 0_{\boldsymbol{n}}\right|+\sum_{\alpha=1}^{M}\left\langle\alpha_{\boldsymbol{n}}\right| f\left(\bar{\varphi}_{\alpha, \boldsymbol{n}}\right)\right) \\
& =\langle\cdot| \prod_{\boldsymbol{n}}\left(1+\sum_{\alpha=1}^{M}\left[f\left(\bar{\varphi}_{\alpha, \boldsymbol{n}}\right)-1\right] \hat{a}_{\alpha, \boldsymbol{n}}^{\dagger} \hat{a}_{\alpha, \boldsymbol{n}}\right)
\end{aligned}
$$


and $f$ is an analytic function of its argument. $F$ is the generating function in that by differentiating $F$ with respect to $f$ and setting $f=1$, we can find all kinds of correlation functions of the particle density. For example,

$$
\left\langle N_{\alpha, \boldsymbol{n}}\right\rangle=\left.\frac{\partial}{\partial f\left(\bar{\varphi}_{\alpha, \boldsymbol{n}}\right)} F(\{\bar{\varphi}\} ; t)\right|_{f=1}=\left\langle\cdot\left|\hat{a}_{\alpha, \boldsymbol{n}}^{\dagger} \hat{a}_{\alpha, \boldsymbol{n}}\right| P ; t\right\rangle .
$$

The exact form of $f$ does not matter. In what follows, we mainly use three kinds of functions,

$$
\begin{aligned}
& f(\bar{\varphi})=\bar{\varphi}, \\
& f(\bar{\varphi})=1+\bar{\varphi}, \\
& f(\bar{\varphi})=\exp (\bar{\varphi}),
\end{aligned}
$$

and these will be used in different contexts.

By differentiating Eq. (15) with respect to $t$, we obtain

$$
\frac{\partial}{\partial t} F(\{\bar{\varphi}\} ; t)=-\langle\{\bar{\varphi}\}|\hat{H}| P ; t\rangle .
$$

Since our main goal is to find a path integral representation of $F(\{\bar{\varphi}\} ; t)$ from Eq. (19), we need to find a linear partial differential equation about $F(\{\bar{\varphi}\} ; t)$. This can be achieved using the following properties of $\langle\{\bar{\varphi}\}|$ :

$$
\begin{aligned}
\langle\{\bar{\varphi}\}| \hat{a}_{\alpha, \boldsymbol{n}}^{\dagger} & =f\left(\bar{\varphi}_{\alpha, \boldsymbol{n}}\right) \hat{V}_{\boldsymbol{n}}\langle\{\bar{\varphi}\}|, \\
\langle\{\bar{\varphi}\}| \hat{a}_{\alpha, \boldsymbol{n}} & =\frac{\partial}{\partial f\left(\bar{\varphi}_{\alpha, \boldsymbol{n}}\right)}\langle\{\bar{\varphi}\}|, \\
\langle\{\bar{\varphi}\}| \hat{a}_{\alpha, \boldsymbol{n}}^{\dagger} \hat{a}_{\beta, \boldsymbol{n}} & =f\left(\bar{\varphi}_{\alpha, \boldsymbol{n}}\right) \frac{\partial}{\partial f\left(\bar{\varphi}_{\beta, \boldsymbol{n}}\right)}\langle\{\bar{\varphi}\}|, \\
\langle\{\bar{\varphi}\}| \hat{a}_{\alpha, \boldsymbol{n}} \hat{a}_{\beta, \boldsymbol{n}}^{\dagger} & =\delta_{\alpha \beta} \hat{V}_{\boldsymbol{n}}\langle\{\bar{\varphi}\}|,
\end{aligned}
$$

where

$$
\hat{V}_{\boldsymbol{n}} \equiv\left(1-\sum_{\beta=1}^{M} \frac{\partial}{\partial \ln f\left(\bar{\varphi}_{\beta, \boldsymbol{n}}\right)}\right)
$$

is the projection operator to the vacuum state at site $\boldsymbol{n}$. Relations (20) yield the partial differential equation for the generating function,

$$
\frac{\partial}{\partial t} F(\{\bar{\varphi}\} ; t)=-\mathcal{L}\left(\{\bar{\varphi}\},\left\{\frac{\partial}{\partial \bar{\varphi}}\right\}\right) F(\{\bar{\varphi}\} ; t),
$$

where $\mathcal{L}$ takes the normal-ordered form, that is, all $\bar{\varphi}$ 's are located to the left side of any $\partial / \partial \bar{\varphi}$. We call $\mathcal{L}$ an evolution operator. Since this is a linear equation, we can write the 
path integral solution of $F(\{\bar{\varphi}\} ; t)$. The path integral solution of Eq. (21) with any of the prescription Eqs. (18) is

$$
F(\{\bar{\xi}\} ; t)=\int \prod_{\alpha, \boldsymbol{n}} \frac{d \eta_{\alpha, \boldsymbol{n}} d \bar{\eta}_{\alpha, \boldsymbol{n}}}{2 \pi i} e^{-\eta_{\alpha, \boldsymbol{n}} \bar{\eta}_{\alpha, \boldsymbol{n}}} F(\{\bar{\eta}\} ; 0) T_{\{\eta\}}^{\{\bar{\xi}\}}(t),
$$

with

$$
T_{\{\eta\}}^{\{\bar{\xi}\}}(t)=\int \mathcal{D}\{\bar{\varphi}\} \mathcal{D}\{\varphi\} e^{-S(\{\bar{\varphi}\},\{\varphi\}, t)+\{\bar{\xi}\} \cdot\{\varphi(t)\}+\{\eta\} \cdot\{\bar{\varphi}(0)\}}
$$

where

$$
S=\int_{0}^{t} d t\left[\sum_{\alpha, \boldsymbol{n}} \bar{\varphi}_{\alpha, \boldsymbol{n}} \frac{\partial}{\partial t} \varphi_{\alpha, \boldsymbol{n}}+\mathcal{L}(\{\bar{\varphi}(t)\},\{\varphi(t)\})\right]
$$

and

$$
\{\bar{\xi}\} \cdot\{\eta\}=\sum_{\alpha, \boldsymbol{n}} \bar{\xi}_{\alpha, \boldsymbol{n}} \eta_{\alpha, \boldsymbol{n}}
$$

Taking a continuum limit and keeping the most relevant terms, we arrive at mesoscopic action which is equivalent to the microscopic master equation. The long-time properties are extracted by studying the action using renormalization-group theory.

\section{EQUIVALENCE BETWEEN STOCHASTIC SYSTEMS}

The equivalence between stochastic systems has been usually studied using similarity transformation for single-species reaction-diffusion systems [22]. This section shows the equivalence between stochastic systems using the evolution equation of the generating function in Eq. (21) with the prescription Eq. (18b) instead of using the similarity transformation. The key mechanism to deduce the equivalence between stochastic systems is the rescaling of the field $\bar{\varphi}$ (see below), which is simple enough to be applicable to higherdimensional systems and multispecies systems.

To begin with, we consider a single-species reaction-diffusion model which is defined on a $d$-dimensional hypercubic lattice with diffusion, pair annihilation, coalescence, death, and single-particle branching. Particles move with a diffusion constant $D$. When two particles form a nearest-neighbor pair, both of them are annihilated with rate $\lambda$ or one of them is removed with rate $\eta$. Additionally, a single particle is annihilated spontaneously with rate $\delta$ and a particle-vacant pair becomes a particle-particle pair with rate $\sigma$. The dynamics are summarized in Table \. When $\delta=\sigma=0$, this model corresponds to the single-species annihilation and coalescence model. The model with $D=\lambda=\eta=0$ is the well-known 
contact process [23]. When all processes are present, this model is known to show an absorbing phase transition which shows the same critical behavior as that of the directed percolation [24].

The Hamiltonian of this reaction-diffusion system is $\hat{H}=\sum_{\boldsymbol{n}}\left(\hat{H}_{\boldsymbol{n}}^{D}+\hat{H}_{\boldsymbol{n}}^{\lambda}+\hat{H}_{\boldsymbol{n}}^{\eta}+\hat{H}_{\boldsymbol{n}}^{\delta}+\hat{H}_{\boldsymbol{n}}^{\sigma}\right)$ with

$$
\begin{aligned}
& \hat{H}_{\boldsymbol{n}}^{D}=D \sum_{i=1}^{d}\left[\hat{\rho}_{\boldsymbol{n}}\left(1-\hat{\rho}_{\boldsymbol{n}+\boldsymbol{e}_{i}}\right)-\hat{a}_{\boldsymbol{n}} \hat{a}_{\boldsymbol{n}+\boldsymbol{e}_{i}}^{\dagger}+\left(1-\hat{\rho}_{\boldsymbol{n}}\right) \hat{\rho}_{\boldsymbol{n}+\boldsymbol{e}_{i}}-\hat{a}_{\boldsymbol{n}}^{\dagger} \hat{a}_{\boldsymbol{n}+\boldsymbol{e}_{i}}\right], \\
& \hat{H}_{\boldsymbol{n}}^{\lambda}=\lambda \sum_{i=1}^{d}\left(\hat{\rho}_{\boldsymbol{n}} \hat{\rho}_{\boldsymbol{n}+\boldsymbol{e}_{i}}-\hat{a}_{\boldsymbol{n}} \hat{a}_{\boldsymbol{n}+\boldsymbol{e}_{i}}\right), \\
& \hat{H}_{\boldsymbol{n}}^{\eta}=\frac{\eta}{2} \sum_{i=1}^{d}\left(2 \hat{\rho}_{\boldsymbol{n}} \hat{\rho}_{\boldsymbol{n}+\boldsymbol{e}_{i}}-\hat{\rho}_{\boldsymbol{n}} \hat{a}_{\boldsymbol{n}+\boldsymbol{e}_{i}}-\hat{a}_{\boldsymbol{n}} \hat{\rho}_{\boldsymbol{n}+\boldsymbol{e}_{i}}\right), \\
& \hat{H}_{\boldsymbol{n}}^{\delta}=\delta\left(\hat{\rho}_{\boldsymbol{n}}-\hat{a}_{\boldsymbol{n}}\right), \\
& \hat{H}_{\boldsymbol{n}}^{\sigma}=\frac{\sigma}{2} \sum_{i=1}^{d}\left[\hat{\rho}_{\boldsymbol{n}}\left(1-\hat{\rho}_{\boldsymbol{n}+\boldsymbol{e}_{i}}\right)-\hat{\rho}_{\boldsymbol{n}} \hat{a}_{\boldsymbol{n}+\boldsymbol{e}_{i}}^{\dagger}+\left(1-\hat{\rho}_{\boldsymbol{n}}\right) \hat{\rho}_{\boldsymbol{n}+\boldsymbol{e}_{i}}-\hat{a}_{\boldsymbol{n}}^{\dagger} \hat{\rho}_{\boldsymbol{n}+\boldsymbol{e}_{i}}\right],
\end{aligned}
$$

where $\hat{\rho}_{\boldsymbol{n}} \equiv \hat{a}_{\boldsymbol{n}}^{\dagger} \hat{a}_{\boldsymbol{n}}$ is the number operator and we drop the unnecessary species index. The operators satisfy the commutation relations Eqs. (11) with $M=1$. Following the procedure explained in Sec. III we can find the evolution operator $\mathcal{L}$ with the prescription Eq. (18b). If we write $\mathcal{L}$ as $\mathcal{L}=\sum_{\boldsymbol{n}} \sum_{i=1}^{d} \mathcal{L}_{\boldsymbol{n}, i}$, we obtain

$$
\begin{aligned}
\mathcal{L}_{\boldsymbol{n}, i} & =(2 D+\delta) \bar{\varphi}_{\boldsymbol{n}} \frac{\partial}{\partial \bar{\varphi}_{\boldsymbol{n}}}-\left(D+\frac{\sigma}{2}\right)\left(\bar{\varphi}_{\boldsymbol{n}} \frac{\partial}{\partial \bar{\varphi}_{\boldsymbol{n}+\boldsymbol{e}_{i}}}+\bar{\varphi}_{\boldsymbol{n}+\boldsymbol{e}_{i}} \frac{\partial}{\partial \bar{\varphi}_{\boldsymbol{n}}}\right) \\
& +\left(\lambda+\frac{\eta}{2}+\frac{\sigma}{2}\right)\left(\bar{\varphi}_{\boldsymbol{n}}+\bar{\varphi}_{\boldsymbol{n}+\boldsymbol{e}_{i}}\right) \frac{\partial^{2}}{\partial \bar{\varphi}_{\boldsymbol{n}} \partial \bar{\varphi}_{\boldsymbol{n}+\boldsymbol{e}_{i}}} \\
& +(\lambda+\eta+\sigma-2 D) \bar{\varphi}_{\boldsymbol{n}} \bar{\varphi}_{\boldsymbol{n}+\boldsymbol{e}_{i}} \frac{\partial^{2}}{\partial \bar{\varphi}_{\boldsymbol{n}} \partial \bar{\varphi}_{\boldsymbol{n}+\boldsymbol{e}_{i}}}+\left(D+\frac{\sigma}{2}\right)\left(\bar{\varphi}_{\boldsymbol{n}}^{2}+\bar{\varphi}_{\boldsymbol{n}+\boldsymbol{e}_{i}}^{2}\right) \frac{\partial^{2}}{\partial \bar{\varphi}_{\boldsymbol{n}} \partial \bar{\varphi}_{\boldsymbol{n}+\boldsymbol{e}_{i}}} \\
& +\frac{\sigma}{2} \bar{\varphi}_{\boldsymbol{n}} \bar{\varphi}_{\boldsymbol{n}+\boldsymbol{e}_{i}}\left[\left(\bar{\varphi}_{\boldsymbol{n}}+\bar{\varphi}_{\boldsymbol{n}+\boldsymbol{e}_{i}}\right) \frac{\partial^{2}}{\partial \bar{\varphi}_{\boldsymbol{n}} \partial \bar{\varphi}_{\boldsymbol{n}+\boldsymbol{e}_{i}}}-\left(\frac{\partial}{\partial \bar{\varphi}_{\boldsymbol{n}}}+\frac{\partial}{\partial \bar{\varphi}_{\boldsymbol{n}+\boldsymbol{e}_{i}}}\right)\right] .
\end{aligned}
$$

If we use an uncorrelated initial condition with density $\rho_{0}$, the initial-state vector and the initial generating function can be written as

$$
\begin{aligned}
|P ; 0\rangle & =\prod_{\boldsymbol{n}}\left[\left(1-\rho_{0}\right)+\rho_{0} \hat{a}_{\boldsymbol{n}}^{\dagger}\right]|0\rangle, \\
F(\{\bar{\varphi}\} ; 0) & =\prod_{\boldsymbol{n}}\left(1+\rho_{0} \bar{\varphi}_{\boldsymbol{n}}\right) .
\end{aligned}
$$

Hence Eqs. (27) and (28) along with Eq. (21) fully specify the above reaction-diffusion system. 
Let us assume that the solution of Eq. (21) is written as $F\left(\{\bar{\varphi}\}, D, \lambda, \eta, \delta, \sigma, \rho_{0} ; t\right)$. Rescaling the field $\bar{\varphi}=\mu \bar{\varphi}^{\prime}(\mu>0), F$ is modified to

$$
F\left(\{\bar{\varphi}\}, D, \lambda, \eta, \delta, \sigma, \rho_{0} ; t\right)=F\left(\left\{\bar{\varphi}^{\prime}\right\}, \tilde{D}, \tilde{\lambda}, \tilde{\eta}, \tilde{\delta}, \tilde{\sigma}, \mu \rho_{0} ; t\right)
$$

where the relations between parameters with and without a tilde are found by setting $\bar{\varphi}=$ $\mu \bar{\varphi}^{\prime}$ in Eq. (27), which read

$$
\begin{aligned}
\tilde{\sigma} & =\mu \sigma, \\
2 \tilde{D}+\tilde{\delta} & =2 D+\delta, \\
\tilde{D}+\frac{\tilde{\sigma}}{2} & =D+\frac{\sigma}{2}, \\
\tilde{\lambda}+\frac{\tilde{\eta}}{2}+\frac{\tilde{\sigma}}{2} & =\frac{1}{\mu}\left(\lambda+\frac{\eta}{2}+\frac{\sigma}{2}\right), \\
\tilde{\lambda}+\tilde{\eta}+\tilde{\sigma}-2 \tilde{D} & =\lambda+\eta+\sigma-2 D .
\end{aligned}
$$

Since $F$ on the right-hand side of Eq. (29) can be regarded as a solution of Eq. (21) with tilded rates and initial density $\mu \rho_{0}$, the two systems connected by Eq. (30) share the same generating function. The relation of the correlation function can be found by differentiating $F$ with $\bar{\varphi}$, which reads

$$
C_{k}\left(\{\boldsymbol{x}\}, D, \lambda, \eta, \delta, \sigma, \rho_{0} ; t\right)=\mu^{-k} \tilde{C}_{k}\left(\{\boldsymbol{x}\}, \tilde{D}, \tilde{\lambda}, \tilde{\eta}, \tilde{\delta}, \tilde{\sigma}, \mu \rho_{0} ; t\right)
$$

where $C_{k}\left(\tilde{C}_{k}\right)$ is the $k$-point correlation functions of the reaction-diffusion systems with the untilded (tilded) transition rates. Thus, for arbitrary $\mu$, which ensures all tilded parameters are non-negative, we can find the equivalent stochastic systems to the system with untilded parameters.

Let us find the equivalent systems to the single-species pair annihilation model $(\eta=\sigma=$ $\delta=0)$. For given $\mu$, the tilded rates are found as

$$
\tilde{D}=D, \tilde{\sigma}=0, \tilde{\delta}=0, \tilde{\eta}=2\left(1-\frac{1}{\mu}\right) \lambda, \tilde{\lambda}=\left(\frac{2}{\mu}-1\right) \lambda,
$$

where $1 \leq \mu \leq 2$ should be satisfied to have the physical meaning. If we choose $\mu=2$ in Eq. (32), the tilded rates become $\tilde{D}=D, \tilde{\eta}=\lambda$, and $\tilde{\delta}=\tilde{\sigma}=\tilde{\lambda}=0$, which are the transition rates of the single-species coalescence model with the initial density $\tilde{\rho}_{0}=2 \rho_{0}$ (of course $\rho_{0}$ should not be larger than $\frac{1}{2}$ ). Hence all kinds of correlation functions of the pair annihilation and coalescence models are related to one another in any dimension. 
This method also reproduces all results in Ref. 22] regarding the equivalence of stochastic systems by adjusting $\mu$ and also finds the initial condition relation.

Next, we apply the generating function method to find the equivalence between threeparticle annihilation models. To our knowledge, this equivalence has not been studied in the literature, although the full renormalization-group study of this model can be found in Ref. 6]. The dynamics of the three-particle annihilation model is summarized in Table [I] The procedure to find the equivalence relations is the same as those explained above. First, we find the Hamiltonian and the corresponding evolution operator. The corresponding Hamiltonian is $\hat{H}=\sum_{\boldsymbol{n}} \sum_{i=1}^{d}\left(\hat{H}_{\boldsymbol{n}, i}^{D}+\hat{H}_{\boldsymbol{n}, i}^{\lambda_{1}}+\hat{H}_{\boldsymbol{n}, i}^{\lambda_{2}}+\hat{H}_{\boldsymbol{n}, i}^{\lambda_{3}}\right)$ with

$$
\begin{aligned}
\hat{H}_{\boldsymbol{n}, i}^{D} & =D\left[\hat{\rho}_{\boldsymbol{n}}\left(1-\hat{\rho}_{\boldsymbol{n}+\boldsymbol{e}_{i}}\right)-\hat{a}_{\boldsymbol{n}} \hat{a}_{\boldsymbol{n}+\boldsymbol{e}_{i}}^{\dagger}+\left(1-\hat{\rho}_{\boldsymbol{n}}\right) \hat{\rho}_{\boldsymbol{n}+\boldsymbol{e}_{i}}-\hat{a}_{\boldsymbol{n}}^{\dagger} \hat{a}_{\boldsymbol{n}+\boldsymbol{e}_{i}}\right] \\
\hat{H}_{\boldsymbol{n}, i}^{\lambda_{1}} & =\frac{\lambda_{1}}{3}\left[3 \hat{\rho}_{\boldsymbol{n}} \hat{\rho}_{\boldsymbol{n}+\boldsymbol{e}_{i}} \hat{\rho}_{\boldsymbol{n}+2 \boldsymbol{e}_{i}}-\hat{a}_{\boldsymbol{n}} \hat{\rho}_{\boldsymbol{n}+\boldsymbol{e}_{i}} \hat{\rho}_{\boldsymbol{n}+2 \boldsymbol{e}_{i}}-\hat{\rho}_{\boldsymbol{n}} \hat{\rho}_{\boldsymbol{n}+\boldsymbol{e}_{i}} \hat{a}_{\boldsymbol{n}+2 \boldsymbol{e}_{i}}-\hat{\rho}_{\boldsymbol{n}} \hat{a}_{\boldsymbol{n}+\boldsymbol{e}_{i}} \hat{\rho}_{\boldsymbol{n}+2 \boldsymbol{e}_{i}}\right], \\
\hat{H}_{\boldsymbol{n}, i}^{\lambda_{2}} & =\frac{\lambda_{2}}{3}\left[3 \hat{\rho}_{\boldsymbol{n}} \hat{\rho}_{\boldsymbol{n}+\boldsymbol{e}_{i}} \hat{\rho}_{\boldsymbol{n}+2 \boldsymbol{e}_{i}}-\hat{a}_{\boldsymbol{n}} \hat{a}_{\boldsymbol{n}+\boldsymbol{e}_{i}} \hat{\rho}_{\boldsymbol{n}+2 \boldsymbol{e}_{i}}-\hat{a}_{\boldsymbol{n}} \hat{\rho}_{\boldsymbol{n}+\boldsymbol{e}_{i}} \hat{a}_{\boldsymbol{n}+2 \boldsymbol{e}_{i}}-\hat{\rho}_{\boldsymbol{n}} \hat{a}_{\boldsymbol{n}+\boldsymbol{e}_{i}} \hat{a}_{\boldsymbol{n}+2 \boldsymbol{e}_{i}}\right] \\
\hat{H}_{\boldsymbol{n}, i}^{\lambda_{3}} & =\lambda_{3}\left[\hat{\rho}_{\boldsymbol{n}} \hat{\rho}_{\boldsymbol{n}+\boldsymbol{e}_{i}} \hat{\rho}_{\boldsymbol{n}+2 \boldsymbol{e}_{i}}-\hat{a}_{\boldsymbol{n}} \hat{a}_{\boldsymbol{n}+\boldsymbol{e}_{i}} \hat{a}_{\boldsymbol{n}+2 \boldsymbol{e}_{i}}\right]
\end{aligned}
$$

and the evolution operator is $\mathcal{L}=\sum_{\boldsymbol{n}} \sum_{i} \mathcal{L}_{\boldsymbol{n}, i}$ with

$$
\begin{aligned}
\mathcal{L}_{\boldsymbol{n}, i} & =D\left(\bar{\varphi}_{\boldsymbol{n}}-\bar{\varphi}_{\boldsymbol{n}+\boldsymbol{e}_{i}}\right)\left(\frac{\partial}{\partial \bar{\varphi}_{\boldsymbol{n}}}-\frac{\partial}{\partial \bar{\varphi}_{\boldsymbol{n}+\boldsymbol{e}_{i}}}\right)+D\left(\bar{\varphi}_{\boldsymbol{n}}-\bar{\varphi}_{\boldsymbol{n}+\boldsymbol{e}_{i}}\right)^{2} \frac{\partial}{\partial \bar{\varphi}_{\boldsymbol{n}}} \frac{\partial}{\partial \bar{\varphi}_{\boldsymbol{n}+\boldsymbol{e}_{i}}} \\
& +\frac{1}{3}\left(\lambda_{1}+2 \lambda_{2}+3 \lambda_{3}\right)\left(\bar{\varphi}_{\boldsymbol{n}}+\bar{\varphi}_{\boldsymbol{n}+\boldsymbol{e}_{i}}+\bar{\varphi}_{\boldsymbol{n}+2 \boldsymbol{e}_{i}}\right) \frac{\partial}{\partial \bar{\varphi}_{\boldsymbol{n}}} \frac{\partial}{\partial \bar{\varphi}_{\boldsymbol{n}+\boldsymbol{e}_{i}}} \frac{\partial}{\partial \bar{\varphi}_{\boldsymbol{n}+2 \boldsymbol{e}_{i}}} \\
& +\frac{1}{3}\left(2 \lambda_{1}+3 \lambda_{2}+3 \lambda_{3}\right)\left(\bar{\varphi}_{\boldsymbol{n}} \bar{\varphi}_{\boldsymbol{n}+\boldsymbol{e}_{i}}+\bar{\varphi}_{\boldsymbol{n}+\boldsymbol{e}_{i}} \bar{\varphi}_{\boldsymbol{n}+2 \boldsymbol{e}_{i}}+\bar{\varphi}_{\boldsymbol{n}+2 \boldsymbol{e}_{i}} \bar{\varphi}_{\boldsymbol{n}}\right) \frac{\partial}{\partial \bar{\varphi}_{\boldsymbol{n}}} \frac{\partial}{\partial \bar{\varphi}_{\boldsymbol{n}+\boldsymbol{e}_{i}}} \frac{\partial}{\partial \bar{\varphi}_{\boldsymbol{n}+2 \boldsymbol{e}_{i}}} \\
& +\left(\lambda_{1}+\lambda_{2}+\lambda_{3}\right) \bar{\varphi}_{\boldsymbol{n}} \bar{\varphi}_{\boldsymbol{n}+\boldsymbol{e}_{i}} \bar{\varphi}_{\boldsymbol{n}+2 \boldsymbol{e}_{i}} \frac{\partial}{\partial \bar{\varphi}_{\boldsymbol{n}}} \frac{\partial}{\partial \bar{\varphi}_{\boldsymbol{n}+\boldsymbol{e}_{i}}} \frac{\partial}{\partial \bar{\varphi}_{\boldsymbol{n}+2 \boldsymbol{e}_{i}}}
\end{aligned}
$$

By rescaling $\bar{\varphi}=\mu \bar{\varphi}^{\prime}$, we find the parameter relations

$$
\begin{aligned}
& \tilde{D}=D, \tilde{\lambda}_{1}+2 \tilde{\lambda}_{2}+3 \tilde{\lambda}_{3}=\mu^{-2}\left(\lambda_{1}+2 \lambda_{2}+3 \lambda_{3}\right), \\
& 2 \tilde{\lambda}_{1}+3 \tilde{\lambda}_{2}+3 \tilde{\lambda}_{3}=\mu^{-1}\left(2 \lambda_{1}+3 \lambda_{2}+3 \lambda_{3}\right), \\
& \tilde{\lambda}_{1}+\tilde{\lambda}_{2}+\tilde{\lambda}_{3}=\lambda_{1}+\lambda_{2}+\lambda_{3} .
\end{aligned}
$$

First consider $3 A \rightarrow 0$ only $\left(\lambda_{1}=\lambda_{2}=0\right)$. Unfortunately, the only possible value of $\mu$ is 1 , which means there is no equivalent procedure to $3 A \rightarrow 0$. However, if we set $\lambda_{2}=\lambda_{3}=0$, we find

$$
\tilde{\lambda}_{1}=\lambda_{1}\left(3-\frac{2}{\mu}\right), \tilde{\lambda}_{2}=\lambda_{1} \frac{-1+4 \mu-3 \mu^{2}}{\mu^{2}}, \tilde{\lambda}_{3}=\lambda_{1}\left(1-\mu^{-1}\right)^{2} \text {, }
$$


where the valid range of $\mu$ is $2 / 3 \leq \mu \leq 1$.

Up to now, we have found the equivalence for single-species reaction-diffusion systems, but the formalism explained above can be extended to multispecies problems. As an example, let us consider a $d$-dimensional two-species annihilation model. There are two kinds of species, $A$ and $B$. Both particles have the same diffusion constant $D$. When two different species form a pair, one of the following reactions may occur. Both particles are removed, or only one particle is annihilated. For the-one particle annihilation process, there are two possibilities. The remaining species stays where it was or moves to the other site. For these dynamics, we assign transition rates $\lambda$ (pair annihilation), $\eta$ (one-particle annihilation without location change), and $\zeta$ (one-particle annihilation with location change). The dynamics is summarized in Table [II.

The Hamiltonian for this model is $\hat{H}=\sum_{\boldsymbol{n}} \sum_{i=1}^{d} \hat{H}_{\boldsymbol{n}, i}$ with

$$
\begin{aligned}
\hat{H}_{\boldsymbol{n}, i}^{D}= & D\left(\hat{A}_{\boldsymbol{n}} \hat{v}_{\boldsymbol{n}+\boldsymbol{e}_{i}}-\hat{a}_{\boldsymbol{n}} \hat{a}_{\boldsymbol{n}+\boldsymbol{e}_{i}}^{\dagger}\right)+D\left(\hat{B}_{\boldsymbol{n}} \hat{v}_{\boldsymbol{n}+\boldsymbol{e}_{i}}-\hat{b}_{\boldsymbol{n}} \hat{b}_{\boldsymbol{n}+\boldsymbol{e}_{i}}^{\dagger}\right) \\
& +D\left(\hat{A}_{\boldsymbol{n}+\boldsymbol{e}_{i}} \hat{v}_{\boldsymbol{n}}-\hat{a}_{\boldsymbol{n}+\boldsymbol{e}_{i}} \hat{a}_{\boldsymbol{n}}^{\dagger}\right)+D\left(\hat{B}_{\boldsymbol{n}+\boldsymbol{e}_{i}} \hat{v}_{\boldsymbol{n}}-\hat{b}_{\boldsymbol{n}+\boldsymbol{e}_{i}} \hat{b}_{\boldsymbol{n}}^{\dagger}\right), \\
\hat{H}_{\boldsymbol{n}, i}^{\lambda}= & \lambda\left(\hat{A}_{\boldsymbol{n}} \hat{B}_{\boldsymbol{n}+\boldsymbol{e}_{i}}-\hat{a}_{\boldsymbol{n}} \hat{b}_{\boldsymbol{n}+\boldsymbol{e}_{i}}+\hat{B}_{\boldsymbol{n}} \hat{A}_{\boldsymbol{n}+\boldsymbol{e}_{i}}-\hat{b}_{\boldsymbol{n}} \hat{a}_{\boldsymbol{n}+\boldsymbol{e}_{i}}\right), \\
\hat{H}_{\boldsymbol{n}, i}^{\eta}= & \eta\left(\hat{A}_{\boldsymbol{n}} \hat{B}_{\boldsymbol{n}+\boldsymbol{e}_{i}}-\hat{a}_{\boldsymbol{n}} \hat{B}_{\boldsymbol{n}+\boldsymbol{e}_{i}}+\hat{A}_{\boldsymbol{n}} \hat{B}_{\boldsymbol{n}+\boldsymbol{e}_{i}}-\hat{A}_{\boldsymbol{n}} \hat{b}_{\boldsymbol{n}+\boldsymbol{e}_{i}}\right. \\
& \left.+\hat{B}_{\boldsymbol{n}} \hat{A}_{\boldsymbol{n}+\boldsymbol{e}_{i}}-\hat{b}_{\boldsymbol{n}} \hat{A}_{\boldsymbol{n}+\boldsymbol{e}_{i}}+\hat{B}_{\boldsymbol{n}} \hat{A}_{\boldsymbol{n}+\boldsymbol{e}_{i}}-\hat{B}_{\boldsymbol{n}} \hat{a}_{\boldsymbol{n}+\boldsymbol{e}_{i}}\right), \\
\hat{H}_{\boldsymbol{n}, i}^{\zeta}= & \zeta\left(\hat{A}_{\boldsymbol{n}} \hat{B}_{\boldsymbol{n}+\boldsymbol{e}_{i}}-\hat{b}_{\boldsymbol{n}}^{\dagger} \hat{a}_{\boldsymbol{n}} \hat{b}_{\boldsymbol{n}+\boldsymbol{e}_{i}}+\hat{A}_{\boldsymbol{n}} \hat{B}_{\boldsymbol{n}+\boldsymbol{e}_{i}}-\hat{a}_{\boldsymbol{n}} \hat{a}^{d} a g_{\boldsymbol{n}+\boldsymbol{e}_{i}} \hat{b}_{\boldsymbol{n}+\boldsymbol{e}_{i}}\right. \\
& \left.+\hat{B}_{\boldsymbol{n}} \hat{A}_{\boldsymbol{n}+\boldsymbol{e}_{i}}-\hat{a}_{\boldsymbol{n}}^{\dagger} \hat{b}_{\boldsymbol{n}} \hat{a}_{\boldsymbol{n}+\boldsymbol{e}_{i}}+\hat{B}_{\boldsymbol{n}} \hat{A}_{\boldsymbol{n}+\boldsymbol{e}_{i}}-\hat{b}_{\boldsymbol{n}} \hat{b}_{\boldsymbol{n}+\boldsymbol{e}_{i}}^{\dagger} \hat{a}_{\boldsymbol{n}+\boldsymbol{e}_{i}}\right),
\end{aligned}
$$

where $\hat{A}_{\boldsymbol{n}}\left(\hat{B}_{\boldsymbol{n}}\right)$ is the number operator for species $A(B)$, and $\hat{v}_{\boldsymbol{n}}$ is the vacuum projection operator with the form $\hat{v}_{\boldsymbol{n}}=1-\hat{A}_{\boldsymbol{n}}-\hat{B}_{\boldsymbol{n}}$. We use a different symbol for the different species and drop the species indices. The commutation relations for the operators in Eq. (37) correspond to the $M=2$ case of Eq. (11). The evolution operator takes the form 
$\mathcal{L}=\sum_{\boldsymbol{n}} \sum_{i=1}^{d} \mathcal{L}_{\boldsymbol{n}, i}$ with

$$
\begin{aligned}
\mathcal{L}_{\boldsymbol{n}, i} & =D\left[\left(\bar{\varphi}_{\boldsymbol{n}}-\bar{\varphi}_{\boldsymbol{n}+\boldsymbol{e}_{i}}\right)\left(\frac{\partial}{\partial \bar{\varphi}_{\boldsymbol{n}}}-\frac{\partial}{\partial \bar{\varphi}_{\boldsymbol{n}+\boldsymbol{e}_{i}}}\right)+\left(\bar{\psi}_{\boldsymbol{n}}-\bar{\psi}_{\boldsymbol{n}+\boldsymbol{e}_{i}}\right)\left(\frac{\partial}{\partial \bar{\psi}_{\boldsymbol{n}}}-\frac{\partial}{\partial \bar{\psi}_{\boldsymbol{n}+\boldsymbol{e}_{i}}}\right)\right] \\
& +D\left[\left(\bar{\varphi}_{\boldsymbol{n}}-\bar{\varphi}_{\boldsymbol{n}+\boldsymbol{e}_{i}}\right)^{2} \frac{\partial}{\partial \bar{\varphi}_{\boldsymbol{n}}} \frac{\partial}{\partial \bar{\varphi}_{\boldsymbol{n}+\boldsymbol{e}_{i}}}+\left(\bar{\psi}_{\boldsymbol{n}}-\bar{\psi}_{\boldsymbol{n}+\boldsymbol{e}_{i}}\right)^{2} \frac{\partial}{\partial \bar{\psi}_{\boldsymbol{n}}} \frac{\partial}{\partial \bar{\psi}_{\boldsymbol{n}+\boldsymbol{e}_{i}}}\right] \\
& +D\left(\bar{\varphi}_{\boldsymbol{n}} \bar{\psi}_{\boldsymbol{n}+\boldsymbol{e}_{i}}+\bar{\psi}_{\boldsymbol{n}} \bar{\varphi}_{\boldsymbol{n}+\boldsymbol{e}_{i}}\right)\left(\frac{\partial}{\partial \bar{\varphi}_{\boldsymbol{n}}} \frac{\partial}{\partial \bar{\psi}_{\boldsymbol{n}+\boldsymbol{e}_{i}}}+\frac{\partial}{\partial \bar{\psi}_{\boldsymbol{n}}} \frac{\partial}{\partial \bar{\varphi}_{\boldsymbol{n}+\boldsymbol{e}_{i}}}\right) \\
& +(D-\eta)\left[\left(\bar{\varphi}_{\boldsymbol{n}}+\bar{\psi}_{\boldsymbol{n}+\boldsymbol{e}_{i}}\right) \frac{\partial}{\partial \bar{\psi}_{\boldsymbol{n}}} \frac{\partial}{\partial \bar{\varphi}_{\boldsymbol{n}+\boldsymbol{e}_{i}}}+\left(\bar{\psi}_{\boldsymbol{n}}+\bar{\varphi}_{\boldsymbol{n}+\boldsymbol{e}_{i}}\right) \frac{\partial}{\partial \bar{\varphi}_{\boldsymbol{n}}} \frac{\partial}{\partial \bar{\psi}_{\boldsymbol{n}+\boldsymbol{e}_{i}}}\right] \\
& +(\zeta+2 \eta+\lambda-d D)\left[\left(\bar{\varphi}_{\boldsymbol{n}}+\bar{\psi}_{\boldsymbol{n}+\boldsymbol{e}_{i}}\right) \frac{\partial}{\partial \bar{\varphi}_{\boldsymbol{n}}} \frac{\partial}{\partial \bar{\psi}_{\boldsymbol{n}+\boldsymbol{e}_{i}}}+\left(\bar{\psi}_{\boldsymbol{n}}+\bar{\varphi}_{\boldsymbol{n}+\boldsymbol{e}_{i}}\right) \frac{\partial}{\partial \bar{\psi}_{\boldsymbol{n}}} \frac{\partial}{\partial \bar{\varphi}_{\boldsymbol{n}+\boldsymbol{e}_{i}}}\right] \\
& +(2 \zeta+2 \eta+\lambda-2 d D)\left(\bar{\varphi}_{\boldsymbol{n}} \bar{\psi}_{\boldsymbol{n}+\boldsymbol{e}_{i}} \frac{\partial}{\partial \bar{\varphi}_{\boldsymbol{n}}} \frac{\partial}{\partial \bar{\psi}_{\boldsymbol{n}+\boldsymbol{e}_{i}}}+\bar{\psi}_{\boldsymbol{n}} \bar{\varphi}_{\boldsymbol{n}+\boldsymbol{e}_{i}} \frac{\partial}{\partial \bar{\psi}_{\boldsymbol{n}}} \frac{\partial}{\partial \bar{\varphi}_{\boldsymbol{n}+\boldsymbol{e}_{i}}}\right),
\end{aligned}
$$

where $\bar{\varphi}(\bar{\psi})$ corresponds to species $A(B)$. By rescaling, we find the relations

$$
\begin{aligned}
& \tilde{D}=D, \quad \tilde{D}-\tilde{\eta}=\frac{1}{\mu}(D-\eta), \\
& \tilde{\zeta}+2 \tilde{\eta}+\tilde{\lambda}-\tilde{D}=\frac{1}{\mu}(\zeta+2 \eta+\lambda-D), \\
& 2 \tilde{\zeta}+2 \tilde{\eta}+\tilde{\lambda}-2 \tilde{D}=2 \zeta+2 \eta+\lambda-2 D,
\end{aligned}
$$

or

$$
\begin{aligned}
& \tilde{D}=D, \quad \tilde{\zeta}=\zeta+\frac{\mu-1}{\mu}(\zeta+2 \eta+\lambda-D), \\
& \tilde{\lambda}=\lambda+\frac{2-2 \mu}{\mu}(\zeta+\eta+\lambda), \quad \tilde{\eta}=\frac{\eta}{\mu}+\frac{\mu-1}{\mu} D .
\end{aligned}
$$

If we set $\eta=\zeta=0$, we can find

$$
\tilde{D}=D, \tilde{\eta}=\frac{\mu-1}{\mu} D, \quad \tilde{\lambda}=\frac{2-\mu}{\mu} \lambda, \quad \tilde{\zeta}=\frac{\mu-1}{\mu}(\lambda-D) .
$$

If $\lambda \geq D$, we obtain equivalent stochastic processes with the pair annihilation model $A+B \rightarrow$

$\emptyset$ for $1 \leq \mu \leq 2$. Other equivalent relations can be found by adjusting $\mu$ for different values of untilded parameters.

\section{SUMMARY AND DISCUSSION}

In summary, we have presented the path integral representation of the generating function for classical stochastic exclusive systems. Using this formalism, we have shown the equivalences of some stochastic systems of single- and two-species models. Furthermore, 
since our formalism is insensitive to the choice of the function $f$ in Eq. (16), we can have different action forms for one system depending on the choice of $f$. Although they may have different forms, the long-time behavior of physical quantities derived from each action form should be the same. For example, if we use the prescription Eq. (18c) in Eq. (16), the action is equivalent to the action with the choice of $f(\bar{\varphi})=\bar{\varphi}$ in Eq. (16) and with the change of fields, such as $\bar{\varphi} \mapsto e^{\bar{\varphi}}$ and $\varphi \mapsto e^{-\bar{\varphi}} \varphi$, performed. In fact, if we use the function $f(\bar{\varphi})=e^{\bar{\varphi}}$ and expand the function $f$ in terms of $\bar{\varphi}$ keeping only terms up to $\bar{\varphi}^{2}$ order, we obtain the same action as the one derived in Ref. [17]. In many cases, any term which contains $\bar{\varphi}$ higher than second order is irrelevant. The method introduced in Ref. [17] is legitimized by the force of the renormalization-group argument. In some cases, usually in reaction-diffusion systems, the canonical dimension of $\bar{\varphi}$ is 0 [], 7], so it is not clear whether we can neglect the higher-order terms after expanding $f$. In this situation, one should use Eq. (18a) or Eq. (18b) to ensure the completeness of the formalism. As an example, let us consider again the model described by the reaction given in Table \with $\eta=\delta=\sigma=0$. If we use Eq. (18b), the action becomes (up to relevant terms)

$$
S=\int d t d^{d} x\left\{\bar{\varphi}\left(\partial_{t}-D \nabla^{2}\right) \varphi+2 \lambda \bar{\varphi} \varphi^{2}+\lambda \bar{\varphi}^{2} \varphi^{2}\right\}
$$

where we neglect the term $D(\nabla \bar{\varphi})^{2} \varphi^{2}$ due to its irrelevance. Equation (42) is exactly the same as the action in Ref. [6] obtained using a bosonic formalism. Since we expect that boson and hard-core boson annihilation models should belong to the same universality class, this coincidence is not surprising. If we perform the change of fields explained above, and keeping terms up to $O\left(\bar{\varphi}^{2}\right)$, the action takes the form

$$
\begin{array}{rl}
S^{\prime}=\int d t d^{d} & x\left\{\bar{\varphi}\left(\partial_{t}-D \nabla^{2}\right) \bar{\varphi}-D \varphi(\nabla \bar{\varphi})^{2}\right. \\
+ & \left.2 \lambda \bar{\varphi} \varphi^{2}-2 \lambda \bar{\varphi}^{2} \varphi^{2}\right\}
\end{array}
$$

which can also be found by the method introduced in Ref. [17]. These two actions, Eq. (42) and Eq. (43), look different, although they describe the same system. This is due to the dropping of higher-order terms of $\bar{\varphi}$. To describe the system properly, we have to keep all the higher-order terms of $\bar{\varphi}$ which are marginal. Then, two actions will give the same long-time behavior of physical quantities.

Although we have concentrated on reaction-diffusion systems, the applicability of this formalism is not restricted to reaction-diffusion systems. We found Langevin equations for DLG 13] and other lattice-gas-type models. This will be published elsewhere [25]. 


\section{Acknowledgments}

This research was supported by the Catholic University of Korea research fund 2004 and Grant No. R05-2003-000-12072-0 from the BRP program of the KOSEF.

[1] B. Chopard and M. Droz, Cellular Automata Modeling of Physical Systems (Cambridge University Press, Cambridge, 1998).

[2] J. Marro and R. Dickman, Nonequilibrium Phase Transitions in Lattice Models (Cambridge University Press, Cambridge, 1999).

[3] M. Doi, J. Phys. A 9, 1465 (1976).

[4] P. Grassberger and M. Scheunert, Fortschr. Phys. 28, 547 (1980).

[5] L. Peliti, J. Phys. (Paris) 46, 1469 (1985).

[6] B.P. Lee, J. Phys. A 27, 2633 (1994).

[7] B.P. Lee and J. Cardy, J. Stat. Phys. 80, 971 (1995).

[8] M.W. Deem and J.-M. Park, Phys. Rev. E 57, 2681 (1998); J.-M. Park and M.W. Deem, ibid. 573618 (1998).

[9] R. Dickman and R. Vidigal, J. Phys. A 35, 7269 (2002); Braz. J. Phys. 33, 73 (2003).

[10] S.A. Janowsky, Phys. Rev. E 51, 1858 (1995).

[11] I. Ispolatov, P.L. Krapivsky, and S. Redner, Phys. Rev. E 52, 2540 (1995).

[12] K.E. Bassler and D.A. Browne, Phys. Rev. E 55, 5225 (1997).

[13] R.K.P. Zia and B. Schmittmann, Phase Transitions and Critical Phenomena, edited by C. Domb and J.L. Lebowitz (Academic Press, New York, 1995), Vol. 17.

[14] V. Brunel, K. Oerding, and F. van Wijland, J. Phys. A 33, 1085 (2000).

[15] P.-A. Bares and M. Mobilia, Phys. Rev. E 59, 1996 (1999).

[16] F. van Wijland, Phys. Rev. E 63, 022101 (2001).

[17] S.-C. Park, D. Kim, and J.-M. Park, Phys. Rev. E 62, 7642 (2000).

[18] S.-C. Park, D. Kim, and J.-M. Park, Phys. Rev. E 65, 015102(R) (2002); S.-C. Park, J.-M. Park, and D. Kim, ibid. 65, 036108 (2002); S.-C. Park and J.-M. Park, ibid. 67, 010103 (2003).

[19] C.W. Gardiner, Handbook of Stochastic Methods, 2nd ed. (Springer-Verlag, Berlin, 1990).

[20] H. Risken, The Fokker-Planck Equation (Springer-Verlag, Berlin, 1984). 
[21] N.G. van Kampen, Stochastic Processes in Physics and Chemistry, enlarged ed. (Elsevier, Amsterdam, 1997).

[22] M. Henkel, E. Orlandini, and G.M. Schütz, J. Phys. A 28, 6335 (1995).

[23] T. E. Harris, Ann. Prob. 2, 969 (1974).

[24] H. Hinrichsen, Adv. Phys. 49, 815 (2000).

[25] S.-C. Park and J.-M. Park (unpublished).

TABLE I: Reaction-diffusion processes of single species and their rates.

\begin{tabular}{ccc}
\hline \hline Diffusion & $A \emptyset \leftrightarrow \emptyset A$ & with rate $D$ \\
Pair annihilation & $A A \rightarrow \emptyset \emptyset$ & with rate $\lambda$ \\
Coalescence & $A A \rightarrow A \emptyset$ & with rate $\eta / 2$ \\
Coalescence & $A A \rightarrow \emptyset A$ & with rate $\eta / 2$ \\
Death & $A \rightarrow \emptyset$ & with rate $\delta$ \\
Branching & $\emptyset A \rightarrow A A$ & with rate $\sigma / 2$ \\
Branching & $A \emptyset \rightarrow A A$ & with rate $\sigma / 2$ \\
\hline \hline
\end{tabular}

TABLE II: Reaction-diffusion processes of the three-particle annihilation model and their rates.

\begin{tabular}{lc}
\hline \hline$A \emptyset \leftrightarrow \emptyset A$ & with rate $D$ \\
$A A A \rightarrow A \emptyset A, \emptyset A A$, or $A A \emptyset$ & with rate $\lambda_{1} / 3$ \\
$A A A \rightarrow A \emptyset \emptyset, \emptyset A \emptyset$, or $\emptyset \emptyset A$ & with rate $\lambda_{2} / 3$ \\
$A A A \rightarrow \emptyset \emptyset \emptyset$ & with rate $\lambda_{3}$ \\
\hline \hline
\end{tabular}


TABLE III: Dynamics of the two-species annihilation model and respective rates.

\begin{tabular}{lr}
\hline \hline$A \emptyset \leftrightarrow \emptyset A$ & with rate $D$ \\
$B \emptyset \leftrightarrow \emptyset B$ & with rate $D$ \\
$A B \rightarrow \emptyset \emptyset$ & with rate $\lambda$ \\
$B A \rightarrow \emptyset \emptyset$ & with rate $\lambda$ \\
$A B \rightarrow A \emptyset$ or $\emptyset B$ & with rate $\eta$ \\
$B A \rightarrow B \emptyset$ or $\emptyset A$ & with rate $\eta$ \\
$A B \rightarrow B \emptyset$ or $\emptyset A$ & with rate $\zeta$ \\
$B A \rightarrow A \emptyset$ or $\emptyset B$ & with rate $\zeta$ \\
\hline \hline
\end{tabular}

